Para enlazar con este artículo / To link to this article:

http://dx.doi.org/10.14198/fem.2017.29.02

Para citar este artículo / To cite this article:

Campoy Aranda, Tomás J., Manuela Balcázar Fines y Viviana Vega Duette. «Los estudiantes universitarios ante la violencia de género». En Marcos Jesús Iglesias Martínez e Inés Lozano Cabezas (coords.), La (in)visibilidad de las mujeres en la Educación Superior: retos y desafios en la Academia. Feminismo/s, 29 (junio 2017): 45-70, DOI: 10.14198/fem.2017.29.02

\title{
LOS ESTUDIANTES UNIVERSITARIOS ANTE LA VIOLENCIA DE GÉNERO
}

\section{THE UNIVERSITY'S STUDENTS AND GENDER VIOLENCE}

\author{
Tomás J. CAMPOY ARANDA \\ tjcampoy@gmail.com \\ orcid.org/0000-0002-1095-8955 \\ Manuela BALCÁZAR FINES \\ mbalcazar203@hotmail.com \\ orcid.org/0000-0001-6873-2871 \\ Viviana VegA DUeTte \\ v.vegaduette@gmail.com \\ orcid.org/0000-0003-3699-5217 \\ Universidad Nacional del Este (Paraguay)
}

\section{Resumen}

El objetivo de este estudio ha sido conocer las creencias sobre igualdad y violencia de género que tienen los estudiantes de la Universidad Nacional del Este (Paraguay), así como determinar las causas y factores de riesgo, con vistas a proponer intervenciones, desde la prevención, eficaces contra el problema, que conlleven a un cambio de la sociedad desde la educación. Realizado en el 2015, abordó como objeto las representaciones y las vivencias que tienen los universitarios sobre la violencia de género, a través de una encuesta aplicada a una muestra de 467 estudiantes de todas las carreras, seleccionados por muestreo aleatorio por conglomerados, de una población de 5678 estudiantes. Los resultados dan cuenta de la presencia de pautas arraigadas en la cultura que podrían hacer persistente la violencia y discriminación hacia la mujer, y de la necesidad de programas que promuevan pautas culturales diferentes que desarraiguen la discriminación y violencia de género.

Palabras clave: género, violencia, igualdad, estudiantes, Universidad.

Feminismo/s 29, junio 2017, pp. 45-70

Los contenidos de la revista se publican bajo una licencia de Creative Commons Reconocimiento 4.0 Internacional (CC BY 4.0) 


\begin{abstract}
The aim of the study has been to learn about the beliefs the student body of the National University of the East (Paraguay) has about equality and gender violence as well as to determine the causes and risk factors so as to propose effective interventions that will help prevent occurrence of the problem and that will bring about change in society through education. The study was carried out in 2015 and it focused on students' representations and experiences regarding gender violence, a survey was conducted among a sample of 467 students from all academic programmes at the university. The sample, from a total population of 5678 students, was put together through random sampling of conglomerates. The findings suggest that there are deep-rooted cultural norms that lead to a persistent violence and discrimination against women and that it is necessary to create programmes that will promote different cultural norms which will eradicate discrimination and gender violence.
\end{abstract}

Keywords: gender, violence, equality, student body, university. 


\section{INTRODUCCIÓN}

La larga historia de desigualdad de la mujer, los avances positivos en este campo, pero al mismo tiempo lenta inserción en la cultura y calidad de vida de la mujer en la sociedad actual, sigue planteando perspectivas de análisis de la violencia de género en los contextos y la necesidad de una intervención más efectiva.

En este sentido Guil Bozal, Solano Parés y Álvarez Girón, señalan que:

A las mujeres se les permite, desde hace tiempo, trabajar en ámbitos públicos y cobrar un sueldo por ello, pero a la hora de tomar decisiones y ocupar los cargos de mayor responsabilidad, salario y prestigio social, se sigue prefiriendo a los varones. Para denominar a este fenómeno, se acuñó en los años ochenta el término «techos de cristal» que define de manera clara la sutileza e invisibilidad del proceso, del que la mayoría de la población, incluso a veces las propias protagonistas, son ajenas. (16)

Desde hace algún tiempo, la presión por la excelencia en el mundo científico y empresarial, unida a la constatación de que son las mujeres quienes tienen hoy en día los mejores expedientes académicos, ha propiciado que desde distintos organismos, tanto públicos como privados, se comience a exigir políticas de igualdad que faciliten a las mujeres la plena incorporación profesional.

También la llegada de las mujeres al mundo del trabajo público ha traído consigo una nueva necesidad aún por atender, que mientras las mujeres permanecieron en el hogar no se detectó; cual es la imprescindible conciliación entre la vida laboral, familiar y personal de hombres y mujeres, pues la gran mayoría se ven obligadas a llevar una doble jornada laboral en casa y fuera de ella.

La asimetría entre los géneros, la desigualdad de oportunidades y de derechos entre estos y el poder de un género sobre otro, aún son incuestionables para muchas personas y entre ellas para muchos docentes de las escuelas o universidades. Las prácticas pedagógicas institucionales a veces, inconscientemente, consolidan relaciones asimétricas.

Cada institución de la sociedad civil y del Estado y sus formaciones culturales: lenguaje, mitos, ideologías, valores, tradiciones, creencias y normas, inciden, influencian, determinan y reproducen las desigualdades entre los 
géneros para mujeres y hombres, en el conjunto de la sociedad y en la cultura (López Safi y Cañete Ribeiro)

Aunque mucho se ha avanzado principalmente en materia formativa y de inserción de la problemática en el debate público, la sociedad actual se caracteriza todavía por una serie de exclusiones aceptadas como «normales» porque forman parte de una cultura arraigada y por tanto imperceptible. Aún no se ha traducido en términos de renta, de igualdad efectiva de derechos y reconocimiento social. En varios países del mundo, las mujeres siguen percibiendo retribuciones menores a igual trabajo que los hombres.

Algunas cifras, como las mencionadas por Ovelar ${ }^{1}$ alertan lo mucho que aún falta hacer en este sentido, pues:

- La ruptura de la servidumbre, aún no ha llevado, ni siquiera en los países más avanzados, al pleno disfrute de los derechos, a la libertad y la igualdad efectivas.

- Dos tercios de los 700 millones de analfabetos del mundo, son mujeres.

- El número de mujeres afectadas por la pobreza rural se ha duplicado en 20 años en el mundo.

- De 1300 millones de pobres extremos que hay en el mundo, el 70\% son mujeres.

- Cada vez más recae sobre la mujer el peso del mantenimiento del hogar, en los países en desarrollo, 1 de cada 3 son hogares encabezados por mujeres solas.

Los roles tradicionales de mujeres y hombres, es decir, de la reproducción de la relaciones desiguales de poder entre los géneros, muchas veces, en la cotidianeidad de las aulas, tanto entre estudiantes, como entre profesoras y profesores, como entre estas/os y aquellas/os y administrativas/os, etc., de manera muy natural. Se entiende aquí que relaciones de poder serían todas aquellas relaciones existentes entre los seres humanos (amorosas, laborales, comunitarias, económicas, pedagógicas, evaluativas, institucionales, investigativas, económicas, políticas, entre otras) en las que unas personas tratan de orientar, conducir e influir en la conducta del otro.

Pero el tema más preocupante, en este contexto, no es solo la discriminación o relación de desventaja, que muchas veces se perciben como normales, sino la violencia.

La violencia de género es una realidad que afecta a un número importante de mujeres. Una parte de ellas aparecen en las estadísticas de víctimas de

1. Blanca Ovelar, Senadora Nacional, ex Ministra de la Educación de Paraguay. Disertación realizada en el III Seminario Taller sobre equidad de género en las Instituciones de Educación Superior, 20 de agosto de 2014. 
violencia de género y otra parte sigue sufriendo cada día situaciones violentas y abusivas que les afectan en diferentes aspectos de sus vidas. Se observa que el tipo de relacionamiento: concubino/a, esposo/a, pareja es el tipo de relacionamiento más frecuente entre víctima y victimario de la violencia (Corte Suprema de Justicia-Secretaría de Género).

En Paraguay, es innegable que hay avances en materia de lucha por la igualdad de género y la no violencia contra la mujer y niños, prueba de ello es la promulgación e implementación de la Ley 1600/2000, contra la Violencia Doméstica, la apertura del Ministerio de la Mujer, y la recientemente aprobada (noviembre del 2016) ley de Protección Integral a las Mujeres contra toda forma de Violencia, entre otros, además de los grandes esfuerzos que desde los diferentes movimientos y grupos de mujeres e instituciones se vienen realizando para luchar por el empoderamiento de la mujer y aminorar el problema de la discriminación y la violencia. Se cuenta con instancias encargadas de atención y prevención, hay un trabajo interinstitucional al respecto, pero los índices de hechos de violencia contra la mujer se mantienen altos y muchas veces con ampliada gravedad.

La violencia intrafamiliar sigue alta y quien más sufre el flagelo es la mujer. El mismo informe del Observatorio de Género de la de la Corte Suprema de Justicia de Paraguay señala que en el año 2014 se registraron 6065 casos de denuncias sobre violencia doméstica y en el año 2015 fueron 6984 casos. Se observa un aumento del 13\%. El número de denuncias de violencia de mujeres sigue siendo 7 veces mayor que el de hombres.

De hecho, la violencia contra las mujeres no puede analizarse ni enfrentarse de manera aislada sino vinculada a los factores de desigualdad económica, social y sobre todo cultural que operan en las relaciones de poder entre hombres y mujeres, como señala CEPAL (ver Informe anual del Observatorio de Igualdad de Género de América Latina y el Caribe).

Además, señala $\mathrm{Casco}^{2}$, la violencia contra las mujeres no es un problema de las mujeres solamente, es de toda la sociedad. Si solo nos concentramos en aumentar la pena y no trabajamos la educación nada va cambiar. Es importante contar con una Ley de Protección a las Mujeres, pero también es hora de instalar el debate en la sociedad y trabajar desde la educación.

2. Rocío Casco, Presidenta de la Comisión de Equidad Social y Género, de la Cámara de Diputados, durante el lanzamiento de la campaña «Por ellas», en setiembre del 2014. 


\section{IGUALDAD Y VIOLENCIA DE GÉNERO}

La violencia de género es un fenómeno que afecta a todas las mujeres de todos los países del mundo, independientemente de la clase social, cultura o edad, y que no se supera con una mayor formación académica (Straus 809). Ya en el año 2004, las Naciones Unidas indicaban que se daba en una de cada tres mujeres en el mundo. En Paraguay, estudios recientes, señalan que una de cada cinco personas sufrió violencia física y el $63 \%$ de ellas no sobrepasa los nueve años de edad.

La Declaración sobre la eliminación de la violencia contra la mujer de las Naciones Unidades, en el artículo I, define que:

Violencia contra la mujer se entiende todo acto de violencia basado en la pertenencia al sexo femenino que tenga o pueda tener como resultado un daño o sufrimiento físico, sexual o sicológico para la mujer, así como las amenazas de tales actos, la coacción o la privación arbitraria de la libertad, tanto si se producen en la vida pública como en la vida privada.

En la misma declaración de la Asamblea General en relación a la violencia de género, en su artículo 2, considera que abarca los siguientes actos:

a) La violencia física, sexual y psicológica que se produzca en la familia, incluidos los malos tratos, el abuso sexual de las niñas en el hogar, la violencia relacionada con la dote, la violación por el marido, la mutilación genital femenina y otras prácticas tradicionales nocivas para la mujer, los actos de violencia perpetrados por otros miembros de la familia y la violencia relacionada con la explotación.

b) La violencia física, sexual y psicológica perpetrada dentro de la comunidad en general, inclusive la violación, el abuso sexual, el acoso y la intimidación sexuales en el trabajo, en instituciones educacionales y en otros lugares, la trata de mujeres y la prostitución forzada.

c) La violencia física, sexual y psicológica perpetrada o tolerada por el Estado, donde quiera que ocurra.

En la actualidad, hay que reconocer los avances que se han producido en los últimos años respecto a la igualdad de oportunidades entre hombres y mujeres, tales como regulación legal, medidas de sensibilización, atención jurídica a las víctimas, investigaciones, etc. No obstante, aún queda mucho camino que recorrer, ya que «la violencia de género ha adquirido proporciones epidémicas» ${ }^{3}$.

La violencia contra las mujeres es una manifestación de las relaciones de poder históricamente desiguales entre mujeres y hombres, que han conducido

3. Margaret Chan, directora general de la OMS, con relación al Informe «Estimaciones mundiales y regionales de la violencia contra la mujer: prevalencia y efectos de la violencia conyugal y de la violencia sexual no conyugal en la salud» de 2013. 
a la dominación masculina, a la discriminación contra las mujeres por parte de los hombres y a impedir su pleno desarrollo. La violencia contra las mujeres tiene su origen en pautas culturales que perpetúan la condición inferior que se asigna a las mujeres en la familia, en el lugar de trabajo, en la comunidad y en la sociedad (Conferencia Mundial de Bejing).

\subsection{La situación de la violencia de género en el contexto internacional}

Según datos de Naciones Unidas, si bien es cierto que el porcentaje de mujeres en los parlamentos se ha casi duplicado en los últimos 20 años, frente a una única mujer que aparecía en dicha lista en 1998; la protección jurídica contra la violencia de género ha aumentado considerablemente, como también otras legislaciones que garantizan la igualdad entre hombres y mujeres; casi todas las regiones en desarrollo han logrado la paridad de género en educación primaria. Sin embargo el progreso ha sido inaceptablemente lento, con estancamientos e, incluso retrocesos, en algunos contextos. Todavía mueren 800 mujeres al día al dar a luz por causas que podrían evitarse. Las mujeres dedican 16 millones de horas al día a buscar agua en países de África Subsahariana (frente a los 6 millones de horas que dedican los hombres). Las mujeres ganan entre un 10 y un 30\% menos que los hombres por realizar el mismo trabajo. Asimismo siguen representando el $60 \%$ de las personas analfabetas a escala mundial. Una de cada tres mujeres sigue sufriendo violencia física o sexual en algún momento de su vida; y, entre 1992 y 2011, solo el 9\% de los negociadores en las mesas de negociación de la paz eran mujeres.

En la Unión Europea (EU), entre las medidas que se vienen aplicando para combatir la violencia contra las mujeres se incluyen la «Directiva sobre la víctimas de delitos» (2012/29/EU) y el «Convenio del Consejo de Europa» (también conocido como Convenio de Estambul) para prevenir y combatir la violencia contra la mujer y la violencia doméstica.

La Agencia de Derechos Fundamentales (FRA) de la EU ha hecho público en 2014, los resultados de una encuesta sobre violencia de género cuyos resultados reflejan que la escalada de violencia contra las mujeres en la UE es grande y que no puede ignorarse. La enormidad del problema prueba que la violencia contra las mujeres no afecta solo a unas pocas.

En 2015, a 20 años de la Conferencia de Beijing, el informe de la ONU de 31 de diciembre de 2015 sobre el empoderamiento de las mujeres y su vínculo con el desarrollo sostenible reconoce que los Estados y otros agentes hoy otorgan gran importancia a la igualdad de género y el empoderamiento de las mujeres, pero que esto no se ve reflejado en la aplicación concreta de políticas ni en la práctica. En casi todos los sectores y regiones persiste la brecha entre 
los géneros y la discriminación contra las mujeres y las niñas. A pesar de los avances educativos que se han dado en la juventud todavía existe una fuerte resistencia al cambio, que es necesario detectar para poder realizar intervenciones que contribuyan a su superación.

El 1 de enero de 2016 entró en vigor la agenda 2030 para el Desarrollo Sostenible que viene a sustituir a la agenda del Milenio y los ODM. La nueva agenda mundial, marcará la senda para construir un mundo más justo y equitativo; entre los que se reconoce la igualdad de género y el empoderamiento de las mujeres, en un objetivo específico y a nivel transversal con metas concretas.

Estudios que se viene realizando sobre la violencia de género dan cuenta sobre la todavía grave situación en este campo, específicamente, en el ámbito universitario. La investigación realizada por Larena y Molina (215), sobre «Violencia de género en las universidades españolas» financiada por el Ministerio de Igualdad/Instituto de la Mujer (Plan Nacional I+D+I, 2004-2007), puso de relieve que la violencia de género, además de ser independiente de la edad, clase social, cultura y nivel académico, también existe en los contexto universitarios.

Asimismo, el «Estudio sobre la juventud universitaria ante la igualdad y la violencia de género» (Universidad Complutense de Madrid) destaca que el sexismo y la violencia de género no son fatalidades biológicas, sino productos culturales, en los que se reproduce un modelo social ancestral por medio de mecanismos que están muy arraigados.

Hay que señalar que se están implementando medidas de prevención e intervención en las universidades europeas, aunque es necesario ampliar actuaciones, pues se trata de que las universidades constituyan un espacio libre de violencia y en avanzar en la construcción de sociedades más igualitarias.

En cuanto a igualdad y violencia de género en el contexto latinoamericano y Caribe, la preocupación de los Estados de la región por erradicar la violencia contra las mujeres ha conducido al logro de diversos e importantes avances en los últimos 20 años. La Comisión Económica para América Latina y el Caribe (CEPAL), con los organismos y las instituciones que conforman el Observatorio: la Organización Panamericana de la Salud (OPS), la Entidad de las Naciones Unidas para la Igualdad de Género y el Empoderamiento de las Mujeres (ONU-Mujeres), el Fondo de Población de las Naciones Unidas (UNFPA), la Secretaría General Iberoamericana (SEGIB) y la Agencia Española de Cooperación Internacional para el Desarrollo (AECID), presentan anualmente un informe de la situación.

Desde el observatorio, se reconocen avances importantes, concentrados en el ámbito del reconocimiento de derechos, las reformas legales y la 
implementación de políticas promisorias; pero la violencia familiar o doméstica y las distintas formas de violencia contra las mujeres, han mostrado la persistencia.

Datos del informe anual de la CEPAL dan cuenta que en el 2013, 88 mujeres fueron asesinadas por sus parejas o ex parejas íntimas en Colombia; 83 en Perú; 71 en República Dominicana; 46 en El Salvador; 40 en Chile; 25 en Uruguay; 20 en Paraguay y 17 en Guatemala. Encuestas muestran que 63 de cada 100 mujeres en México y 60 de cada 100 mujeres en Ecuador han padecido algún incidente de violencia, ya sea por parte de su pareja u otra persona. Esta violencia ocurre con mayor frecuencia entre quienes tienen o han tenido pareja (casadas, unidas, separadas, divorciadas o viudas): 48,7\% en Ecuador, 47\% en México y 45,4\% en Uruguay.

Pese al avance normativo, la Comisión Económica para América Latina y el Caribe (CEPAL) advierte sobre la falta de políticas públicas e instituciones capaces de asegurar su efectiva aplicación en los países. En este sentido, recomienda mejorar la producción de información sobre la violencia de género; llevar a cabo, cuando sea necesario, procesos de reforma legislativa con debates participativos; lograr una coordinación institucional fuerte, de alta jerarquía y gran capacidad técnica; e instaurar un sistema verdaderamente integral de atención, capaz de articular las políticas sociales con las respuestas judiciales.

Asimismo para su prevención y erradicación no bastan únicamente cambios legislativos y presupuestarios, pues hace falta un enfoque integral del problema. Son necesarias medidas de protección a corto plazo acompañadas de un trabajo sostenido para una transformación cultural y un cambio de conciencia en todos los ámbitos.

Entre las sugerencias emanadas de la consulta, se recomienda a América Latina abordar las causas estructurales de la desigualdad y la discriminación de género; línea en la que se enmarca esta investigación, considerando que la persistencia de la desigualdad real, se debe a muchos factores, pero en especial a factores culturales.

Es en este contexto donde la educación en general y, en especial, la Educación Superior, debe establecer estrategias integras y de fuerza suficiente para apuntar al cambio cultural en este sentido. Pero muchas veces, en el seno de la universidad latinoamericana, se repite la misma socialización en relaciones de género que hay fuera de sus paredes y hasta se conserva aún una fuerte estructura jerárquica que favorece relaciones desiguales. 


\subsection{La igualdad y violencia de género en Paraguay}

En cuanto a la violencia de género Bott, Guedes, Goodwin y Mendoza (6) señalan que la violencia física y sexual contra las mujeres es un problema aún muy extendido en América Latina; Paraguay, está entre los 10 países de la región con más violencia de género. Más del 20 por ciento de las paraguayas ha sufrido alguna vez violencia doméstica, según un estudio realizado a 180 mil mujeres en 12 países de la región. Pero como advierte Guedes ${ }^{4}$, los datos podrían ser incluso mayores ya que en ocasiones el miedo a represalias en el hogar o a que la información pueda filtrarse lleva a las mujeres a no denunciarlo. Entre las mujeres que indicaron haber sufrido algún tipo de violencia, entre el 28 y el 64 por ciento dijeron que nunca había buscado ayuda, ni había contado su situación a nadie por vergüenza, por miedo a represalias o no saber dónde ir. El estudio destaca que es posible prevenir la violencia contra la mujer. Sin embargo, todos los entornos informaron de violencia contra la mujer y que la prevalencia variaba según el entorno, lo cual indica que los altos niveles de violencia no son una característica inevitable de la sociedad.

Los datos también revelan que la raíz de la violencia de las mujeres está en los papeles desiguales de género, en sociedades que promueven la sumisión de la mujer y la dominación por parte del hombre, por lo que cualquier acción, según los expertos, requiere promover la igualdad de género y el fomentar el papel de la mujer.

Diferentes estudios (CEPEC, 2012; Bott, Guedes, Goodwin y Mendoza; CEDAW, 2011), recomiendan en este sentido:

- Trabajar con miras a transformar instituciones utilizando una perspectiva de género; en particular integrar la atención a la violencia contra la mujer.

- Promover el empoderamiento social y económico de mujeres, niñas y niños.

- Involucrar a hombres y niños varones para promover la no violencia y la equidad de género.

- Concienciar a actores clave del ámbito de la niñez y adolescencia para realizar acciones de prevención, identificación, seguimiento y denuncia de casos de abuso sexual en la niñez a través de medidas tales como

4. Asesora regional de violencia interfamiliar para la Organización Panamericana de la Salud (OPS) y coautora del estudio sobre Violencia contra la mujer en América Latina y el Caribe, en entrevista con Efe, durante la presentación del libro en 2014. 
capacitaciones, campañas y difusión de información con enfoque de derechos y género.

- Impulsar la visualización de la violencia contra las mujeres y de género como problemas sociales con consecuencias individuales colectivas, médicas, legales y de derechos humanos.

- Buscar generar cambios en la visión sobre las relaciones de género orientadas a la equidad, la igualdad y la no violencia a través del sistema de educación formal como campañas que enfoquen los derechos humanos

- Intensificar esfuerzos por aumentar la concienciación entre los funcionarios públicos, especialmente las fuerzas del orden, los funcionarios judiciales, los proveedores de salud y los trabajadores sociales, y refuerce la concienciación de que esa es una violencia social y moralmente inaceptable, una violación de sus derechos humanos.

La violencia de género, la violencia doméstica y la violencia contra la mujer por parte de su pareja, a pesar de todas las normativas y acuerdos aprobados, sigue en aumento en el Paraguay.

Estudios recientes, como la Primera Encuesta sobre Violencia Intrafamiliar basada en Género-Área Urbana (Ministerio de la Mujer 103-108), señala en su informe final que encontró, entre otros:

- $\quad 80,5 \%$ de las mujeres sufrieron violencia psicológica intrafamiliar más de una vez, y el 88,9\% sufrieron violencia física intrafamiliar «más de una vez».

- 6 de cada 10 personas que sufrieron violencia viven en pareja.

- De las personas que sufrieron violencia física, según la persona que le agredió, prevalece la pareja como el esposo/a o compañero/a permanente (que suma el 53\% de los casos), le sigue en tercer lugar el/la ex esposo/a con el 11,9\%.

- De las personas que sufrieron violencia psicológica según la persona que le agredió nuevamente se visualiza al esposo/a o compañero/a permanente en $53,6 \%$, levemente superior a lo registrado en la violencia física.

- 1 de cada 5 mujeres afirmó que estaba embaraza cuando sufrió violencia física o psicológica.

- 7 de cada 10 personas que sufrieron violencia física no denunciaron, y 9 de cada 10 personas que sufrieron violencia psicológica tampoco lo hicieron. 
A más de estudios, como el citado precedentemente, y los índices demarcados por el Observatorio de la Corte Suprema de Justicia sobre casos denunciados en juzgados de Paz del país, que reflejan aumento de las denuncias de hechos de violencia doméstica e intrafamiliar; unos pocos titulares de periódicos nacionales, de los últimos años, muestran que la violencia de género tal vez se acrecienta.

Informaciones recogidas de la presa paraguaya señalan que hubo casi 90 mujeres asesinadas en cuatro años. De las 86 mujeres asesinadas entre 2011 y 2015 en Paraguay, en situaciones de violencia doméstica, 66 fueron ultimadas a manos de sus concubinos, esposos o ex concubinos (ABC Color: 29 de enero 2016). Entre 2010 y mediados de 2015, un total de 359 mujeres fueron asesinadas en diferentes circunstancias en Paraguay, un país con una cultura marcadamente machista. A su vez, el Ministerio de la Mujer atendió, sólo el año pasado, 1.877 casos de violencia (ABC Color: 28 de enero 2016). El 80\% de las consultas que se realizan en el servicio de Gineco-Obstetricia del Hospital de Clínicas, revela que las mujeres han sufrido algún tipo de violencia de género (ABC Color, 25 de noviembre 2014). Tan solo en el Ministerio de la Mujer se reportaron en 2013 un total de 2267 casos de violencia contra la mujer, cifra que revela crecimiento con respecto a años anteriores, según se expresa en estadísticas (HOY, 8 de Enero 2014).

Estas citas muestran que en Paraguay la violencia contra la mujer es aún una lacra cotidiana y que merece atención desde diferentes ámbitos y que el contexto descrito, brevemente, requiere atención de la justicia y la educación. Principalmente este último, pues según Bourdieu (Lamas 45) sostiene que el orden social masculino está tan profundamente arraigado que no requiere justificación, se impone a sí mismo y es tomado como natural gracias al acuerdo casi perfecto e inmediato que obtiene de las estructuras sociales y la división sexual del trabajo, y, por otra, de las estructuras cognitivas inscritas en los cuerpos y en las mentes.

Los conceptos de género estructuran la percepción y la organización concreta y simbólica de toda la vida social. La diferenciación a partir del género ubica a la mujer en una posición de desventaja, discriminándola en base a su rol reproductivo, que es tomado como fundamento para naturalizar y justificar su subordinación y opresión. Las diferentes funciones esperadas de los varones y las mujeres no son vistas como algo aprendido a través del proceso de socialización y la educación, sino como características naturales e innatas de cada sexo.

Respecto de la inserción femenina en el escenario de la Universidad, Casimiro-Soriguer (37-54) considera que las mujeres se incorporan 
abiertamente al campo laboral, lo que hace emerger el dilema de la igualdad en el mundo de lo público, pero que lo desplaza al mundo de lo privado.

Sin duda el planteamiento de la igualdad y la representación de hombres y mujeres en el espacio público presenta dificultades, fundamentalmente porque si partimos del principio de que las personas somos complejas en nuestra individualidad y diversas en las formas de socialización, es imposible imaginar un tratamiento exactamente igual.

En el escenario de la Academia este debate cobra un singular dinamismo, pues en un contexto centrado en ser equitativo e igualitario desde el discurso, algunas acciones evidencian desequilibrios entre los sexos, tales como la segregación de las carreras, la ausencia relativa de las mujeres en los órganos de toma de decisiones y sobre todo, la exclusión o tal vez autoexclusión, priorizando a su carrera, la atención de los requerimientos familiares, sociales y otros.

\section{MÉTODO}

El estudio de carácter descriptivo, enfoque cuantitativo, ex post-facto, tipo encuesta, se realizó en el contexto de la Universidad Nacional del Este (UNE, Paraguay), durante 2015.

\subsection{Participantes}

La población estuvo constituida por estudiantes de las 7 facultades y las 25 carreras, de la que se ha extraído una muestra representativa mediante muestreo probabilístico, estratificado, aleatorio proporcional.

Tabla 1. Participantes

\begin{tabular}{|l|l|}
\hline \multicolumn{2}{|c|}{ FICHA TÉCNICA } \\
\hline Población & 5678 \\
\hline Muestra invitada & 374 \\
\hline Muestra productora & 467 \\
\hline Diseño de muestreo & Muestreo aleatorio por conglomerados \\
\hline Nivel de confianza & $95 \%$ \\
\hline
\end{tabular}

El porcentaje de hombres ha sido de $43,5 \%$ y el de mujeres 56,5 (media 1,44 y una $\sigma$ de, 496 . La edad media ha sido de 22,97, rango de 40 años (edad mínima 17 y máxima 57). 
Tabla 2. Frecuencia de hombres y mujeres por carreras y cursos

\begin{tabular}{|c|c|c|c|c|c|}
\hline FACULTAD & CURSO & TOTAL & TOTAL & HOMBRES & MUJERES \\
\hline \multirow{2}{*}{$\begin{array}{l}\text { INGENIERÍA } \\
\text { AGRONÓMICA }\end{array}$} & Ing. Agrónoma & 16 & \multirow{2}{*}{25} & 7 & 9 \\
\hline & Ing. Ambiental & 9 & & 4 & 5 \\
\hline \multirow{3}{*}{$\begin{array}{l}\text { CIENCIAS } \\
\text { ECONÓMICAS }\end{array}$} & Administración & 24 & \multirow{3}{*}{127} & 12 & 12 \\
\hline & Contabilidad & 80 & & 27 & 53 \\
\hline & Economía & 23 & & 8 & 15 \\
\hline \multirow{7}{*}{ FILOSOFÍA } & CC. Educación & 5 & \multirow{7}{*}{84} & 2 & 3 \\
\hline & Psicología & 47 & & 14 & 33 \\
\hline & CC. Comunicación & 15 & & 7 & 8 \\
\hline & Letras & 2 & & 1 & 1 \\
\hline & Matemática & 11 & & 5 & 6 \\
\hline & Filosofía & 2 & & 1 & 1 \\
\hline & Historia & 2 & & 1 & 1 \\
\hline \multirow{4}{*}{ POLITÉCNICA } & $\begin{array}{l}\text { Análisis de } \\
\text { sistemas }\end{array}$ & 24 & \multirow{4}{*}{98} & 18 & 6 \\
\hline & Ingen. Eléctrica & 36 & & 29 & 7 \\
\hline & Lic. en turismo & 14 & & 1 & 13 \\
\hline & Ing. de sistemas & 24 & & 15 & 9 \\
\hline \multirow{3}{*}{$\begin{array}{l}\text { DERECHO } \\
\text { Y CIENCIAS } \\
\text { SOCIALES }\end{array}$} & Derecho & 68 & \multirow{3}{*}{85} & 31 & 37 \\
\hline & CC. Políticas & 5 & & 2 & 3 \\
\hline & Notariado & 12 & & 3 & 9 \\
\hline \multirow{2}{*}{$\begin{array}{l}\text { CIENCIAS DE } \\
\text { LA SALUD }\end{array}$} & Medicina & 27 & \multirow{2}{*}{39} & 8 & 19 \\
\hline & Enfermería & 12 & & 3 & 9 \\
\hline \multirow{4}{*}{$\begin{array}{l}\text { ESCUELA } \\
\text { SUPERIOR DE } \\
\text { BELLAS ARTES }\end{array}$} & Artes Visuales & 3 & \multirow{4}{*}{9} & 1 & 2 \\
\hline & Danza & 2 & & 1 & 1 \\
\hline & Música & 2 & & 1 & 1 \\
\hline & Teatro & 2 & & 1 & 1 \\
\hline & & & TOTAL & 203 & 264 \\
\hline
\end{tabular}

\subsection{Instrumento de recolección de datos}

Para la recogida de la información se ha elaborado la «Escala sobre Igualdad y Prevención de la Violencia de Género». Este instrumento en la redacción definitiva consta de 28 ítems a los que se ha sometido a un análisis factorial. El valor de KMO es ,842 y sig. ,000. El método de rotación ha sido varimax. 
El análisis de componentes principales nos indica que el porcentaje total de la varianza explicada es de 54,65 para 4 factores; el gráfico de sedimentación también nos sugiere un total de 4 dimensiones. El primer factor «Percepción de la mujer» consta de 8 ítems, el segundo factor «Relación de pareja» (dominio y sumisión) consta de 8 ítems, el tercer factor «Relación de pareja» (como reacción) consta de 8 ítems y el cuarto factor «Relaciones de convivencia» consta de 4 ítems. La fiabilidad del instrumento (consistencia interna alfa de Cronbach) es ,830.

Las variables generales (sexo, edad, carrera, curso, estado civil, tener hijos, trabajo remunerado, estudios padre, estudios madre, se han sometido a la prueba de bondad de ajuste, cuya Significalidad estadística se recoge en el siguente cuadro.

Tabla 3. Significalidad estadística

\begin{tabular}{|c|c|c|c|c|c|}
\hline \multirow{2}{*}{ Sexo } & Asimetría & Curtosis & Variable & Prueba binomial & $\mathrm{P}$ valor \\
\hline &, 257 & $-1,942$ & discreta & 7,627 &, 007 \\
\hline \multirow{2}{*}{ Edad } & Asimetría & Curtosis & Variable & K-S & $P$ valor \\
\hline & , 104 & $-1,405$ & discreta & 10,070 &, 000 \\
\hline \multirow{2}{*}{ Carrera } & Asimetría & Curtosis & Variable & Chi-cuadrado & $\mathrm{P}$ valor \\
\hline & , 104 & $-1,405$ & discreta & 539,479 &, 000 \\
\hline \multirow{2}{*}{ Curso } & Asimetría & Curtosis & Variable & Chi-cuadrado & $\mathrm{P}$ valor \\
\hline & 1,092 & ,845 & discreta & 666,488 &, 000 \\
\hline \multirow{2}{*}{ Estado civil } & Asimetría & Curtosis & Variable & Chi-cuadrado & P valor \\
\hline & 2,983 & 9,551 & discreta & 1315,024 &, 000 \\
\hline \multirow{2}{*}{ Hijos } & Asimetría & Curtosis & Variable & Prueba binomial & $\mathrm{p}$ valor \\
\hline & $-2,110$ & 2,465 & discreta & 244,234 &, 000 \\
\hline \multirow{2}{*}{ Trabajo } & Asimetría & Curtosis & Variable & Prueba binomial & $\mathrm{P}$ valor \\
\hline &,- 073 & $-2,003$ & discreta & 619 & ,459 \\
\hline \multirow{2}{*}{$\begin{array}{l}\text { Estudios } \\
\text { Padre }\end{array}$} & Asimetría & Curtosis & Variable & Chi-cuadrado & $\mathrm{P}$ valor \\
\hline &, 004 & $-1,269$ & discreta & 16,484 &, 000 \\
\hline \multirow{2}{*}{$\begin{array}{l}\text { Estudios } \\
\text { Madre }\end{array}$} & Asimetría & Curtosis & Variable & Chi-cuadrado & $\mathrm{P}$ valor \\
\hline &,- 034 & $-1,323$ & discreta & 10,469 &, 000 \\
\hline
\end{tabular}

\subsection{Análisis de los datos}

Una vez realizada la prueba de bondad de ajuste se determinaron los estadísticos a aplicar para establecer las diferencias significativas. Para ello se ha 
aplicado la prueba de chi-cuadrado con la finalidad de observar si los datos siguen una distribución uniforme. La prueba de Kruskal-Wallis para determinar las medianas de los grupos que difieren cuando tienen datos que no son simétricos (estadística no paramétrica).

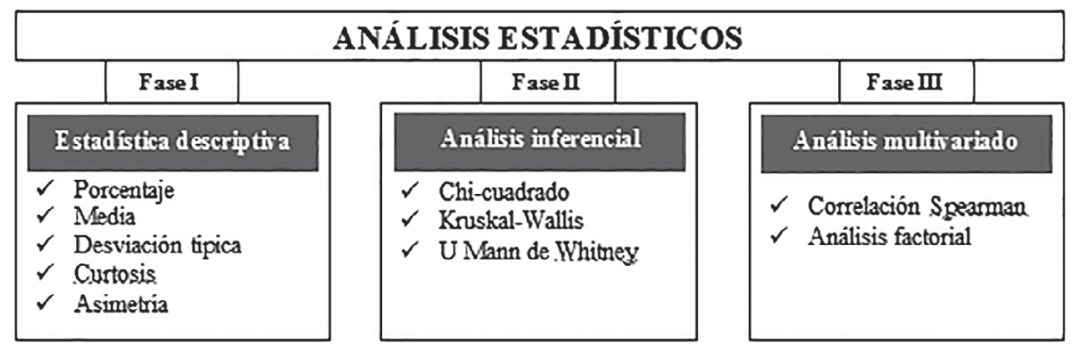

Figura 1. Funciones estadísticas utilizadas

\section{RESULTADOS}

\subsection{Percepción de la mujer}

Esta dimensión ha tenido por objetivo conocer creencias respecto a las mujeres y su rol en la sociedad y qué conductas de las mismas son consideradas «adecuadas» o no. Como resultados en esta dimensión, en base al análisis de los ítems que lo conforman, se ha obtenido:

\section{DIMENSIÓN I: PERCEPCIÓN DE LA MUJER}

V17. Las mujeres intentan ganar poder controlando a los hombres.

V18. En el fondo, las mujeres feministas pretenden que la mujer tenga más poder que el hombre.

V19. Las mujeres feministas están haciendo demandas completamente irracionales a los hombres.

V20. Con el pretexto de pedir «igualdad» las mujeres buscan privilegios especiales, tales como condiciones de trabajo que favorezcan a ellas sobre los hombres.

V21. Las mujeres interpretan comentarios o conductas inocentes como sexistas, es decir, como expresiones de prejuicio o discriminación contra ellas.

V22. Las mujeres exageran los problemas que tienen en el trabajo.

V23. Existen mujeres que, para burlarse de los hombres, primero se insinúan sexualmente a ellos y luego rechazan los avances de éstos.

V24. Las mujeres no aprecian lo que los hombres hacen por ellas.

Figura 2. Ítems de la Dimensión Percepción de la Mujer 


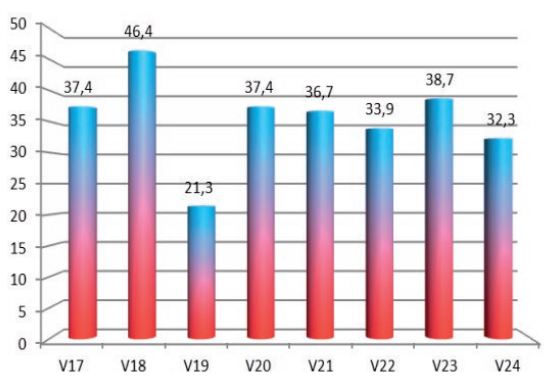

Figura 3. Percepción de la mujer paraguaya

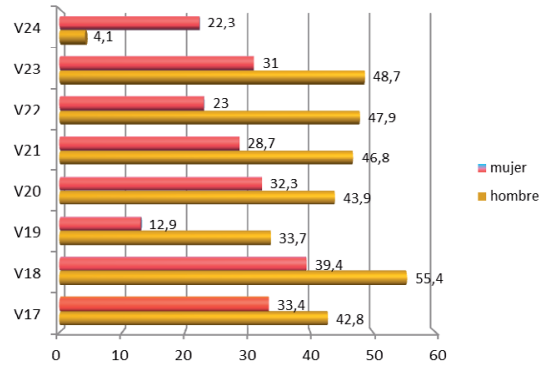

Figura 4. Percepción de la mujer pya (por sexo)

El 37,4\% de los estudiantes piensa que las mujeres intentan ganar poder controlando a los hombres. Hay diferencias significativas en función del sexo (chi-cuadrado, p-valor=, 050). Pero no hay diferencias significativas en función del nivel de estudios de los padres, lo que puede indicar hasta qué punto está todavía enraizada la visión tradicional que se tiene de la mujer en la sociedad paraguaya. Esta opinión está más presente en los estudiantes más jóvenes en las edades comprendidas entre 17 y 20 años (Kruskal-Wallis, sig.,009) y en los solteros (chi-cuadrado, p-valor $=, 004$ ).

El 46,4\% de los estudiantes considera que lo que pretenden las mujeres feministas es que la mujer tenga más poder. En función del sexo (chi-cuadrado, p-valor $=, 001)$, los hombres $(55,4 \%)$ están más de acuerdo que las mujeres $(39,4 \%)$. La edad también es una variable que establece diferencias (KruskalWallis, sig.,002), en especial, los estudiantes más jóvenes. También el estado civil es una variable a considerar, pues los solteros coinciden más (chi-cuadrado, p-valor=,037). El 30,2\% de los estudiantes cree que las feministas hacen demandas irracionales a los hombres. Hay un 39,9\% que no tiene clara su respuesta. Hay diferencias en función del sexo (chi-cuadrado, p-valor $=, 000$ ) y la edad (Kruskal-Wallis, sig. =007). El 33,7\% de los hombres así lo considera frente al $12,9 \%$ de las mujeres.

El $37,4 \%$ de los estudiantes piensa que las mujeres con el pretexto de pedir igualdad buscan privilegios. El $68,2 \%$ no tiene opinión. Hay diferencias significativas en función del sexo, el 43,9\% de los hombre así lo cree frente al $32,3 \%$ de las mujeres (chi-cuadrado, p-valor=,037). Por estado civil, los solteros $(38,7 \%)$ son los que más coinciden (chi-cuadrado, p-valor=,021). El $36,7 \%$ de los encuestados considera que las mujeres exageran los comentarios 
o conductas sexistas. Hay un $33 \%$ que no tiene respuesta clara. Hay diferencias significativas en función del sexo (chi-cuadrado, p-valor=,000 y el estado civil ,039). Uno de cada tres encuestados (33,9\%) piensa que las mujeres exageran los problemas que tienen en el trabajo. Hay un $29,7 \%$ de indecisos. Se dan diferencias significativas en función del sexo (chi-cuadrado, p-valor=,000); estudios del padre (p-valor=,011), estudios de la madre (p-valor=,010). El $38,7 \%$ de los estudiantes piensa que hay mujeres que se insinúan sexualmente a los hombres para luego rechazarlos. Hay diferencias significativas en función del sexo (chi-valor, p-valor=,000) y la edad (Kruskal-Wallis, sig. ,033). Los hombres creen que las mujeres se insinúan $(48,7 \%)$, pero llama la atención que el $31 \%$ de la mujeres también lo piensan.

\subsection{Relaciones de pareja (dominio y sumisión)}

Una de las manifestaciones del sexismo es la tendencia a legitimar las relaciones de dominio/sumisión entre hombre y mujer, de manera que se convierte en factor de riesgo. En las relaciones de pareja primero se llega al dominio y sumisión, y después al control. El control se puede ejercer sobre cualquier aspecto de la autonomía de la mujer a la que se busca subordinar. A continuación se recogen los ítems considerados en esta dimensión para analizar los resultados obtenidos:

\footnotetext{
DIMENSIÓN II: RELACIONES DE PAREJA (DOMINIO Y SUMISIÓN)

V25. Las personas no pueden ser felices en sus vidas a menos que tengan pareja.

V26. Por bien de tus hijos/as, aunque la mujer tenga que soportar la violencia de su pareja, conviene que no lo denuncie.

V27. Está justificado que un hombre agreda a su pareja cuando ella decide dejarle.

V28. Para tener una buena relación de pareja es deseable que la mujer evite contrariar al hombre.

V29. Cuando una mujer es agredida por su pareja algo habrá hecho para provocarle.

V30. La violencia que se produce dentro de la casa es un asunto de la familia y no debe salir de ahí.

V31. Si una mujer es maltratada por su pareja y no le abandona será porque no le disgusta del todo esta situación.

V32. Está bien que los hombres salgan con muchas mujeres, pero no al revés.
}

Figura 5. Ítems de la Dimensión de Relaciones de Pareja 


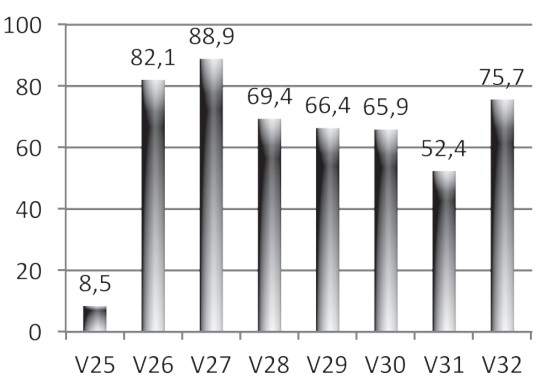

Figura 6. Relación de pareja

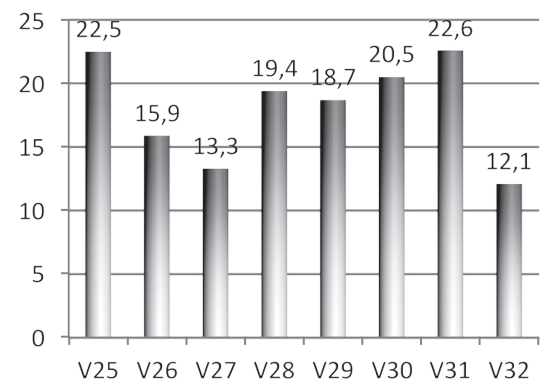

Figura 7. Relación de pareja (indecisos)

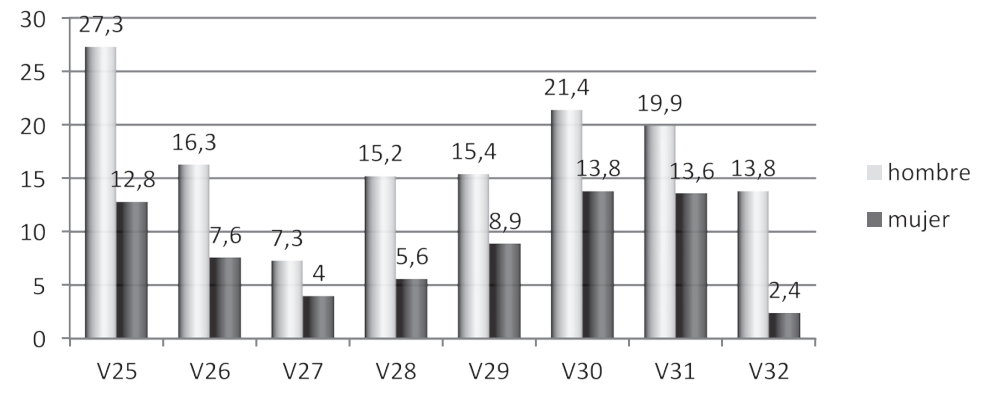

Figura 8. Diferencias en el grado de acuerdo por sexo

Algo más de la mitad de los estudiantes $(55,4 \%)$ no cree necesario tener pareja para ser felices. Hay un 22,5\% de indecisos. Hay diferencias significativas en función del sexo (chi-cuadrado, p-valor=,000). Son los hombres $(27,3 \%)$ los que más sienten la necesidad de tener pareja para ser felices que las mujeres $(12,8 \%)$. Uno de cada cinco encuestados $(11,4 \%)$ es partidario de que por el bien de los hijos la mujer tiene que soportar la violencia sin que haya denuncia; el $82,1 \%$ es partidario de presentar denuncia. Los hombres son más partidarios de que no se produzca ninguna denuncia (16,3\%) que las mujeres $(7,6 \%)$. El $5,6 \%$ estudiantes piensa que está justificado que el hombre agreda a su pareja cuando ella decide dejarlo. Los hombres están más de acuerdo $(7,3 \%)$ que las mujeres (4\%). El 11,8\% de los estudiantes justifica la violencia de género, es decir, piensa que cuando una mujer es agredida por su marido es porque algo habrá hecho. Hay diferencias en función de la edad (Kruskal-Wallis, sig.,025), pues los más jóvenes son los que más justifican esta actitud. Asimismo el porcentaje de hombres que no tiene una opinión clara es $29,1 \%$ y el de mujeres $16,2 \%$. Son madres con estudio universitarios las que más justifican esta actitud $\mathrm{y}$ los de nivel de estudio primario las que menos (chi-cuadrado, p-valor=,050). 
El 17,2\% de los estudiantes opina que la violencia que se produce dentro de la casa es un asunto de familia y que no se debe dar a conocer. Hay diferencias significativas en relación a la edad (Kruskal-Wallis, sig.,020). Los más partidarios de guardar silencio son los más jóvenes. El 16,7\% de los estudiantes piensa que si una mujer es maltratada por su compañero y no le abandona será porque no le disgusta del todo esta situación. Hay un alto porcentaje de indecisos $(31,4 \%)$. El porcentaje de hombres que justifica esa actitud de la mujer es 19,9 y el de las mujeres 13,6.

En relación a si está bien que los hombres salgan con muchas mujeres, pero no al revés, hay un 17,2\% que está de acuerdo. Hay diferencias en relación a los estudios de la madre (chi-cuadrado, p-valor=,002), pues son las mujeres con mayor nivel de estudios la que están más a favor.

\subsection{Relaciones de pareja (como reacción)}

Los estereotipos de género no sólo se mantienen sino que se agudizan en algunos casos. Aunque son las mujeres las que más han cambiado en casi todos los aspectos, los valores de libertad y autonomía personal se han impuesto y chocan con el estereotipo femenino tradicional que frena los avances en igualdad. Las cualidades masculinas más valoradas tienen que ver con el poder, la fuerza, la valentía o la firmeza; mientras que en las mujeres son la sensibilidad, la capacidad de perdón y la generosidad.

A continuación los Ítems que forman parte de esta dimensión:

\begin{tabular}{|l|}
\hline \multicolumn{1}{|c|}{ DIMENSIÓN III: RELACIÓN DE CONVIVENCIA (COMO REACCIÓN) } \\
\hline V33. Los hombres deben proveer seguridad económica a las mujeres. \\
V34. Un buen padre debe hacer saber a su familia quién es el que manda. \\
V35. El hombre que parece agresivo es más atractivo. \\
V36. Está justificado agredir a alguien que te ha quitado lo que era tuyo. \\
V37. Es correcto pegar a alguien que te ha ofendido. \\
V38. Para tener una buena relación de pareja conviene que el hombre sea un poco \\
$\quad$ superior a la mujer, en edad, en el dinero que gana, en la toma de decisiones. \\
V39. Hay que disculpar los celos porque son una expresión de amor. \\
V40. Si alguien te pega, pégale tú. \\
\hline
\end{tabular}

Figura 9. Ítems de la Dimensión de Relación de Convivencia

En opinión de los estudiantes, solo el 23,7\% de los encuestados no está de acuerdo con que los hombres deben proveer económicamente a las mujeres. El porcentaje de mujeres que está en descuerdo es 37,3 y el de hombres 52,9.

El $72,9 \%$ de los estudiantes considera que un buen padre debe dejar claro a su familia quien es el que manda. Hay diferencias significativas en función 
del sexo (chi-cuadrado, p-valor=,000) y de la edad (Kruskal-Wallis, sig. 001). Los hombres son más partidarios de hacer saber a su familia quién es el que manda $(18,5 \%)$ que la mujer $(7 \%)$.

El porcentaje de estudiantes que no está de acuerdo con que un hombre que muestra conductas agresivas resulta más atractivo es 5,0. Un 13,4\% de indecisos. Hay diferencias significativas en relación al sexo (chi-cuadrado, p-valor=,000), edad (Kruskal-Wallis, sig. 015) y estado civil (chi-cuadrado, p-valor=,036). El hombre está más de acuerdo $(6,8 \%)$ que la mujer $(3,6 \%)$. En los hombres el 22,5\% no tiene postura.

El 8,2\% de los estudiantes piensa que se justifica agredir a alguien que te ha quitado algo. El $17,1 \%$ de los encuestados no tiene postura. Hay diferencias significativas en función del sexo (chi-cuadrado, p-valor=,000), de la edad (Kruskal-Wallis, sig. 001) y el nivel de estudios del padre (chi-cuadrado, p-valor $=, 021)$. Los hombres $(11,2 \%)$ manifiestan una actitud más agresiva que las mujeres (5,8\%). Además hay diferencias en cuanto a la opción de indecisos (25,5\% los hombres y 10,4\% las mujeres). La agresividad se manifiesta más en los estudiantes más jóvenes, solteros y cuyos padres tienen hasta estudios secundarios.

El 7,4\% de los estudiantes considera que es correcto pegar a alguien que te ha ofendido. El porcentaje de indecisos es 16,9\%. Se establece diferencias en función del sexo (chi-cuadrado, p-valor=,000). El 13,8\% de los hombres está más de acuerdo con la conducta de agresión que las mujeres (2,4\%). El $12,8 \%$ de los estudiantes piensa que para tener una buena relación de pareja conviene que el hombre sea superior a la mujer. Hay diferencias en función del sexo (chi-cuadrado, p-valor=,002) y de la edad (Kruskal-Wallis, sig. 001). El porcentaje de mujeres que están «muy en desacuerdo» es 55,8\% y el de hombres es $38,5 \%$. Algo más de la mitad de los estudiantes rechaza que los celos son una expresión de amor motivo por el que hay que disculparlos $(55,6 \%)$. Además hay un 26,5\% que no tiene una postura clara. El porcentaje de mujeres que está de acuerdo $(12,5)$ es menor que el de hombres $(24,9)$ (chi-cuadrado, p-valor $=, 001)$.

El 18,2\% de los estudiantes opina que si recibe una agresión hay que devolverla. El 17,2\% no tiene opinión. Las mujeres están menos de acuerdo en devolver la agresión (12,1\%) que los hombres (25,8\%) (chi-cuadrado, p-valor $=, 034$ ).

Del amplio análisis de los resultados se deduce que:

- En general, entre un 25\% y un 30\% de los estudiantes no rechaza la violencia de género y reconoce el «poder» del hombre sobre la mujer. 
- Las mujeres rechazan más el poder y jerarquía del hombre.

- Hay un porcentaje alto de estudiantes que no tienen opinión formada sobre las cuestiones que se plantean (oscila entre el 13\% y el 31\%).

- Los estudiantes más jóvenes (entre 17 y 20 años) mantienen posturas más extremas y justifican más las conductas violentas.

- El 44,4\% de los estudiantes no rechaza los celos porque son una expresión de amor.

\subsection{Relaciones de convivencia}

Las relaciones interpersonales están presentes en todos los ámbitos en que nos manejemos, siendo un aspecto básico y esencial de nosotros. La convivencia constituye uno de los aspectos más importantes en las relaciones humanas. Como manifestación natural de estas relaciones pueden surgir desavenencias, desacuerdos, comunicación inadecuada, etc., que pueden dar lugar a conflictos interpersonales y derivar en conductas violentas.

Los ítems de este factor analizado son los que siguen:

\begin{tabular}{|l|}
\hline \multicolumn{1}{|c|}{ DIMENSIÓN IV: RELACIONES DE CONVIVENCIA } \\
\hline 41. Las mujeres deben ser queridas y protegidas por los hombres. \\
\hline $\begin{array}{l}\text { 42. Si alguien quiere pelearse contigo, trata de convencerle de que hay otra forma de } \\
\text { resolver los problemas. }\end{array}$ \\
\hline 43. Si alguien me insulta, lo ignoro. \\
\hline 44. Una buena relación de pareja debe establecerse de igual a igual. \\
\hline
\end{tabular}

Figura 10. Ítems de la Dimensión Relaciones de Convivencia

Los resultados de nuestro estudio indican:

El 83,0\% de los estudiantes está de acuerdo con que las mujeres deben ser queridas y protegidas por los hombres. Hay un 10,2\% de indecisos. Hay diferencias significativas en función de la edad (Kruskal-Wallis, sig.,003). Los estudiantes a partir del $3 .^{\circ}$ año son los que más coinciden con el ítem.

El 87,2\% de los estudiantes afirma que es mejor ignorar a alguien que te insulta. Hay un $17,1 \%$ que no tiene una postura clara. No hay diferencias significativas entre las variables de agrupación y los distintos ítems.

Cuatro de cada cinco estudiantes $(81,0 \%)$ piensa que si alguien quiere pelearse hay que hacer ver que hay otra forma de resolver los problemas 


\section{CONCLUSIONES}

El objetivo general del estudio ha sido conocer, a través de la opinión del alumnado universitario, las creencias del mismo, con relación a la igualdad y a la violencia de género, considerando qué percepción tiene de la mujer; cómo son las relaciones de pareja, en relación al dominio y sumisión; las relaciones de pareja, como reacción; y las relaciones de convivencia.

Con relación a la percepción sobre la mujer, se concluye que:

- «Sombrea» sobre los hombres un pensamiento machista.

- Persisten ideas tales como que las mujeres intentan controlar a los hombres, piden privilegios, y exageran sus problemas.

- Hay una creencia extendida de que a las mujeres les gusta insinuarse sexualmente a los hombres, para luego rechazarlos.

- Casi un tercio piensa que las mujeres están agradecidas por lo que los hombres hacen por ellas.

En cuanto al factor relaciones de pareja (dominio y sumisión):

- Los hombres sienten más necesidad de tener pareja que las mujeres.

- El 30,6\% considera que las mujeres deben dar la razón al hombre para una buena relación de pareja.

- Un tercio de los estudiantes no rechaza que el hombre agreda a la mujer.

- Un tercio de los estudiantes justifica la violencia doméstica y que hay que guardar silencio.

- Casi la mitad de los estudiantes $(48,1 \%)$ no rechaza la actitud de maltrato hacia la mujer.

- El 34,1\% de los estudiantes no ve mal que un hombre pueda salir con varias mujeres.

En la dimensión de relaciones de pareja, como reacción:

- Las mujeres rechazan más el poder y jerarquía del hombre.

- Un porcentaje significativo (hasta el 31\%) de los estudiantes no tienen una opinión formada sobre los temas que se plantean.

- Los estudiantes más jóvenes (17-20 años) justifican más las conductas violentas

- Cerca de la mitad de los estudiantes $(44,4 \%)$ no rechaza los celos porque son una expresión de amor. 
Con referencia al objetivo relacionado al tratamiento de igualdad y violencia de género:

- La igualdad y prevención de la violencia de género recibe escasa atención en la UNE. Se trata a lo sumo de alguna actividad puntual, pero no existe una visión amplia sobre estos temas que se concrete en acciones planificadas y coordinadas, orientadas a toda la comunidad educativa.

- Los estudiantes demandan charlas informativas sobre estos temas. Sólo un porcentaje reducido considera que se debe trabajar desde la formación.

Con relación a conocer las creencias sobre igualdad y violencia de género que tiene el alumnado de la Universidad Nacional del Este, se destaca que los resultados obtenidos muestran que hay una relación de poder del hombre sobre la mujer, el 30\% de los hombres reconoce el poder y la jerarquía de éste sobre la mujer, el 46,4\% de los hombres piensa que las mujeres buscan dominar al hombre. Hay porcentajes preocupantes donde se llega a justificar la violencia ejercida sobre la mujer, incluso siendo más acusado en los estudiantes más jóvenes. No rechazan que el hombre agreda a la mujer cuando ésta decida dejarlo y un 48,1\% no rechaza la violencia sobre la mujer.

Como causas y factores de riesgo resultan preocupantes los aspectos culturales con pautas arraigadas, por las que muchas de las formas de violencia contra la mujer son minimizadas o tomadas como normales, no generan reacción de los estudiantes. Al contrario, se manifiesta una especie de valoración del silencio - no denuncia - de la mujer como forma de evitar más violencia, o el de soportar la violencia por el bien de sus hijos. En general, hay una tendencia a mantener una postura pasiva, de no denuncia, de mal menor y conformismo.

El pensamiento machista persiste $(44,4 \%)$. Los estudiantes no rechazan totalmente los celos porque son una expresión de amor y tampoco ven mal que un hombre pueda salir con varias mujeres. Incluso los estudiantes más jóvenes justifican determinadas conductas machistas.

Como factor de riesgo, también se evidencia la escasa atención o la insuficiencia de las actividades realizadas en el contexto universitario para la generación de un cambio cultural, y tal vez en todo el sistema educativo nacional; pues si los mismos estudiantes universitarios muestran escasa conciencia con relación a la gravedad de la violencia contra la mujer, es un indicativo de que estos temas no se han abordado suficientemente en su proceso formativo.

Los resultados evidencian que son los estudiantes más jóvenes quienes justifican o están de acuerdo con determinadas conductas machistas, por lo que se considera de importancia para promover la igualdad y prevenir la violencia 
de género reforzar los programas de intervención desde la educación, en la etapa adolescente.

\section{REFERENCIAS BIBLIOGRÁFICAS}

Benítez, Aldo. «La violencia contra la mujer en números». ABC Color: Asunción, Paraguay. 28 de enero 2016.

Benítez, Aldo y Flavia Borja. «Hubo casi 90 mujeres asesinadas en cuatro años». ABC Color: Asunción, Paraguay. 29 de enero 2016.

Bott, Sara, Alessandra Guedes, Mary Goodwin y Jennifer Adams Mendoza. «Violencia contra la mujer en América Latina y el Caribe», 2013. <http://www.paho.org/ hq/index.php?option=com_docman\&task=doc_view\&gid=21425\&Itemid > consultado el 12-10-2014.

Bourdieu, Pierre. «La Lógica de los Campos». Revista Zona Erógena 16 (1993). <http://es.scribd.com/doc/127889702/bourdieu-pierre-la-logica-de-los-campos-entrevista>, consultado el 03-06-2014.

Casimiro-Soriguer, Milagrosa. «Las mujeres en la Ciencia». La ausencia de las mujeres en los contenidos escolares. (2004): 37-54. 12 de octubre de 2014.

CEPAL, FAO, ONU Mujeres, PNUD, OIT «Trabajo decente e igualdad de género. Políticas para mejorar el acceso y la calidad del empleo de las mujeres en América Latina y el Caribe», 2013. <http://www.ilo.org/wcmsp5/groups/public/ americas/--ro-lima/--sro-santiago/documents/publication/wcms_233161.pdf>, consultado el 12-10-2014.

Comisión Económica para América Latina y el Caribe (CEPAL). «Informe anual 2013-2014. El enfrentamiento de la violencia contra las mujeres en América Latina y el Caribe», 2014. <http://repositorio.cepal.org/bitstream/ handle/11362/37185/S1500499_es.pdf, consultado el 24-05-2014

Corte Suprema de Justicia - Observatorio de Género. «Violencia doméstica e Intrafamiliar 2014 y 2015, denunciados en Juzgados de Paz del país», 2015. <www.pj.gov.py/contenido/136-secretaria-de-genero/609>, consultado el 16-01-2016.

Diario $\mathrm{ABC}$ «Consultas revelan violencia». $A B C$ Color: Asunción, Paraguay. 25 de noviembre 2014

Diario Hoy. «El macho paraguayo sigue pegando: solo 7 mujeres por día se atreven a denunciarlo». HOY: Asunción, Paraguay 8 de Enero 2014.

Guil Bozal, Ana, Ana Solano Parés y Manuela Álvarez Girón. La Situación de las Mujeres en las Universidades Públicas Andaluzas. Sevilla: Consejo Económico y Social de Andalucía, 2005.

Lamas, Marta. La perspectiva de género una herramienta para construir equidad entre mujeres y hombres. México: Sistema integral para el Desarrollo de la Familia (DIF), México, 1997. 
Larena, Rosa y Silvia Molina. «Violencia de género en las universidades: Investigaciones y medidas para prevenirla». Revista de Investigaciones en Intervención Social, 1.2 (2010): 202-219.

Ministerio de la Mujer, Centro Superior de Estudios de Administración y Finanzas. «Primera Encuesta Sobre Violencia Intrafamiliar Basada en Género-Área Urbana, 2014. <http://www.mujer.gov.py/index.php/publicaciones>, consultado el 15-03-2016.

Naciones Unidas. Declaración sobre la eliminación de la violencia contra la mujer, 1993. <http://www.ohchr.org/SP/ProfessionalInterest/Pages/ViolenceAgainstWomen. aspx>, consultado el 12-08-2014.

Naciones Unidas. Informe Anual 2014-2105 de ONU Mujeres, 2015. <http:// www.unwomen.org/es/digital-library/publications/2015/6/annualreport-2014-2015>, consultado el 07-11-2015.

Naciones Unidas. Informe de la Cuarta Conferencia Mundial sobre la Mujer, 1996. <http://www.un.org/womenwatch/daw/beijing/pdf/Beijing\%20full\%20 report\%20S.pdf>, consultado el 04-06-2014.

Straus, Murray A. (2004) «Prevalence of violence against Dating Partners by Male and Female University Students Worldwide». Violence Against Women, 10.7 (2004): 790-811. 12 de octubre de 2014.

Universidad Complutense de Madrid, Ministerio de Sanidad, Servicios Sociales e Igualdad. «La juventud universitaria ante la igualdad y la violencia de género», 2013. <http://www.upm.es/sfs/Rectorado/Gerencia/Igualdad/Documentos/ Juventud_Universitaria_ante_igualdad_y_violencia_de_genero_\%202012. pdf>, consultado el 12-10-2014. 